This is the pré-publication version of the paper published on Technoetic Arts journal.

Final paper reference:

de Souza e Silva, A. (2004). From simulations to hybrid space: how nomadic technologies change the real. Technoetic Arts: an international journal of speculative research, 1 (3), 209-221.

\title{
FROM SIMULATIONS TO HYBRID SPACE how nomadic technologies change the real
}

\section{Adriana de Souza e Silva}

\begin{abstract}
This paper states that the concept of real is modified by the emergence of nomadic technology devices, which are responsible for creating a hybrid reality that merges physical and digital spaces. The concept of virtual space is analyzed from the perspective of arts and science fiction. The first section shows how the concept of virtual space as a mindspace has been developed. The idea of cyberspace as a place for the mind emphasizes the traditional Cartesian doubt, that is, does the mental image correspond to the real? Second, the paper argues that the concept of the virtual changes, since it can no longer be considered disjoint from physical space; rather, it belongs to it. Finally, the movies The Thirteenth Floor (Rusnak 1999) and The (Matrix Wachowski 1999) are case studies that illustrate how the idea of inhabiting a virtual space has changed from the traditional notion of virtual space as a place for the mind to a hybrid space that is part of our lives. As a result, it amplifies the notion of what the real can be.
\end{abstract}

Keywords: hybrid space, physical, virtual, interfaces, real, computer simulations 


\section{INTRODUCTION}

In the last few years we observed a change in what was formerly called cyberspace. [1] At first considered an immaterial space, built for the human mind and detached from physical reality, now it merges with the physical environment. Mainly due to the emergence of mobile technology devices, ubiquitous computing, and transparent interfaces, we live in a hybrid reality, which includes both the virtual and the physical. The goal of this paper is to argue that the ubiquitousness caused by new digital technologies changes and enlarges the concept of what the real can be. Consequently, it generates a new relationship between the real and the imaginary.

With the emergence of the World Wide Web in the 1990s, we observed the growth of a new type of space, a virtual digital realm that shared with physical space the status of reality. Hence, it expanded our ways of inhabiting the world, because virtual spaces could also be built according to our imagination. Inhabiting imaginary spaces is not a novelty in human history. Arts and literature have for a long time filled the human mind with new and "unreal" places. Nevertheless, there has never been a virtual space with which people could interact via diverse interfaces. In addition, it has become possible to communicate with people from different places in the same virtual environment, thus creating a new sense of community.

Cyberspace, however, has been traditionally considered an immaterial space, a place for the mind, contrasting with physical reality, inhabited by the physical body. The perception of cyberspace as a mindspace, and the strict separation between physical and virtual have contributed to create a whole imaginary realm in science fiction. Movies like The Matrix and The Thirteenth Floor, which are going to be analyzed later, are some 
examples of how cyberspace has been envisioned as a simulation space which one could inhabit. The emergence of this new virtual space, which was viewed as non-physical, although real, addressed again the traditional Cartesian doubt, that is, does the mental image correspond to reality?

In the last 50 years, the development of computer simulations and virtual reality allowed the creation of other types of realities that could also be imaginary, that is, that might not exist in the physical world. The opportunity to inhabit and interacting with these alternative realities has changed the traditional distinction between real and imaginary. Henceforth, the imaginary was not only considered what was inside our minds but also virtual spaces that could be constructed by means of technology and that can be shared with other people. Nowadays new mobile computer technologies generate a new relationship between the real and the imaginary. How was this distinction formerly addressed?

\section{WHAT IS REAL? THE CONSTRUCTION OF VIRTUAL/MIND SPACE}

\section{II.1. Descartes and the distinction between the real and the imaginary}

When Descartes challenged the connection between mental image and external reality, he created the psychological concept of the idea. The philosopher was interested in exploring whether the mental image corresponded to the physical world and even if there was a physical world at all (or if everything was just a product of our minds). Doubting everything but the thought, Descartes questioned whether our perception of the 
real matches reality. Are we aware of the totality of the real surrounding us? Or is what we call "real" no more than a simulation of the real? In this case, what is the real? How can we know that anything that is mental represents anything that is not mental? Or better: does the mind represent the world?

The possibility of creating a representation of the external world inside our minds, one that could have no connection at all with reality, has greatly contributed to the development of the concept of cyberspace as a mindspace and as a place that could be disconnected from the physical world.

\section{II.2. Cyberspace as a mindspace: immateriality and simulation}

Placing cyberspace inside the mind emphasizes its disconnection from the physical space and addresses it as another type of reality: an alternative place for our minds to inhabit and a space in which one could play with different selves.

The vision of cyberspace as a place for the mind has been widely explored in literature, especially in science fiction since William Gibson's Neuromancer (Gibson 2000) in 1985. Gibson creates the concept of cyberspace as a place which can be inhabited by downloading one's mind into an information space. For him it is a dataspace, which one could inhabit through neural implants. Also, this place represented a space of liberty, since users perceived themselves as no longer attached to the "weight" of their physical bodies. During the 1990s, especially after the emergence of the World Wide Web, cyberspace has been viewed as another (and generally better) place, disconnected from the physical world. 
Critical for the construction of this concept of virtual space are specific interfaces used to connect to it: a computer screen and cables connected to the telephonic network. This situation has two main consequences. First, in order to connect to the Internet it is necessary to disconnect from the physical world. It is necessary to stop moving in order to enter the Internet. Second, the screen as an interface represents a "barrier" in the relationship between virtual and physical spaces. The need for avatars is a consequence of this fact. Since one cannot be physically in cyberspace, there is the need to represent the subject (through avatars) in the digital space. That is why body issues have always been critical for the development of multiuser environments. Once in cyberspace, the user is disconnected from her physical body and therefore able to create as many bodies as she desires. Consequently, issues about multiple identities have become a central point of study during the last decade (Turkle 1995). In addition, science fiction stories like Neuromancer, which have contributed to the conceptualization of digital space, generally show the connection to cyberspace through neural implants which require the user's immobility.

Since the connection with cyberspace was disconnected from physical (material) space, virtual space began to be considered immaterial. In addition, since the physical is considered real, cyberspace became a place for the imaginary. The possibility of creating virtual reality environments in which the user could feel immersed but could not actually touch anything also contributed to the immateriality of cyberspace. But how can a space created by means of physical interfaces be considered immaterial? The connection to the Internet has always been made through the use of a keyboard, a mouse and a screen: material interfaces. The Internet itself is a network of physical computers, working as 
servers and routers, connected by wires and waves. Also, most of VR-immersive environments use the Head Mounted Display (HMD) as an interface.

William Gibson's description of cyberspace as a "consensual hallucination" emphasized virtual space as a place that existed inside the mind, as well as an information space. Information has historically been regarded as an immaterial entity, disconnected from any materiality. However, according to Katherine Hayles, "for information to exist, it must always be instantiated in a medium" (Hayles 1999: 13). Therefore, it is impossible to disconnect information from the physical artifacts that embody it and make it real. Also, cyberspace would never be possible without physical computers and networks.

Consequently, for users who may not know the material processes involved, the impression is that information is predominant over materiality, since it is frequently thought that information can manifest itself on several different material supports and still be the same. However, more important than the actual information is the medium that carries it, since the physical support has the ability to change the meaning of the message. This means that information can never be detached from the material supports (or interfaces) that carry it. Hence, it is not possible to disconnect matter and information. There could never be an information space without the physical interfaces that actualize it.

Since Descartes, we have become accustomed to the dualism that separates mind and body, immaterial and material. From this dualistic point of view, mind and immateriality are generally considered better and more pure than matter. In addition to the philosophical tradition, an important fact that contributed to the concept of cyberspace as a mindspace was the development of computers as simulation machines. 
From its inception, the function of a computer has been to simulate real life processes. According to William Flake (Flake 2000: 5), "one of the first uses of computers was to simulate the evolution of complicated equations." Later, computers were used to represent weather simulations, economic models, and cognitive models of the brain. Hence, we can conclude that computers were born as simulation machines, even before the invention of the graphic user interface (GUI), which was intended to simulate on the screen the physical space of the desk. According to Sherry Turkle (Turkle 1995), the graphic interface's opacity that emerged with the desktop metaphor marks a division line between a culture of calculations and a culture of simulations. The GUI is intended to simulate our physical desk and, thus, create a closer relationship between computers and humans. Henceforth, we started to consider computers as spatial entities, as long as we could interact with them. Similarly, Janet Murray (Murray 1999) affirms that computers started to be seen as space when it was first possible to interact with them. From this perspective, the graphic interface is not responsible for the digital environment's spatiality; it only emphasizes it.

Besides simulating space, computers are also able to simulate life. Therefore, it is possible to argue that computers were created to simulate both the world as well as the world's inhabitants. Also in the 1970s, John Conway invented the Game of Life, based on John von Neumann's study of cellular automata (CAs). In the 1940s, von Neumann envisaged a systematic theory which would be mathematical and logical in form, and which would contribute in an essential way to our understanding of natural systems (natural automata) as well as to our understanding of both analog and digital computers (artificial automata). Neumann wanted to study reproduction abstractly, but the word 
"cellular" is not meant biologically when used in this context. It refers, rather, to adjacent spaces that form a pattern. Interestingly, he concentrated on the simplest mathematical framework that would allow information to reproduce. CAs were not invented, therefore, to be realistic models of nature, but to represent information reproduction.

Three decades later, Conway simplified von Neumann's ideas creating a pattern where cells could be either alive or dead (on or off), and that had a very simple set of rules for determining what the next state of the system would be. The game is played on a two-dimensional grid. Each cell has eight neighbors, adjacent across the sides and corners of the square.

Computers have been used to simulate life, and they may also create whole new forms of life. A frequent question among artists and professionals who build virtual worlds and environments has been whether virtual spaces and creatures should simulate their physical correspondents or be developed with no reference at all to reality. Why should we simply reproduce physical creatures and spaces in the digital space of computers, if there is the possibility to experiment with whole new types of spaces and life forms? Due to the fact that cyberspace was viewed as a dataspace and information is considered immaterial, digital life and spaces could in theory assume any form they wanted.

For example, Karl Sims' Evolved Virtual Creatures (MIT 1994) is a computer simulation that deals with the Darwinian evolution of virtual block creatures. Virtual agents living in a 3D virtual environment are programmed to evolve on their own through competition with other beings. Each one has different skills, such as swimming, flying, or crawling. Sims has also created simulations with evolving virtual plants. In Panspermia 
(1990), artificial evolution techniques were used to interactively select from random mutations of plant shapes until a variety of interesting structures emerged.
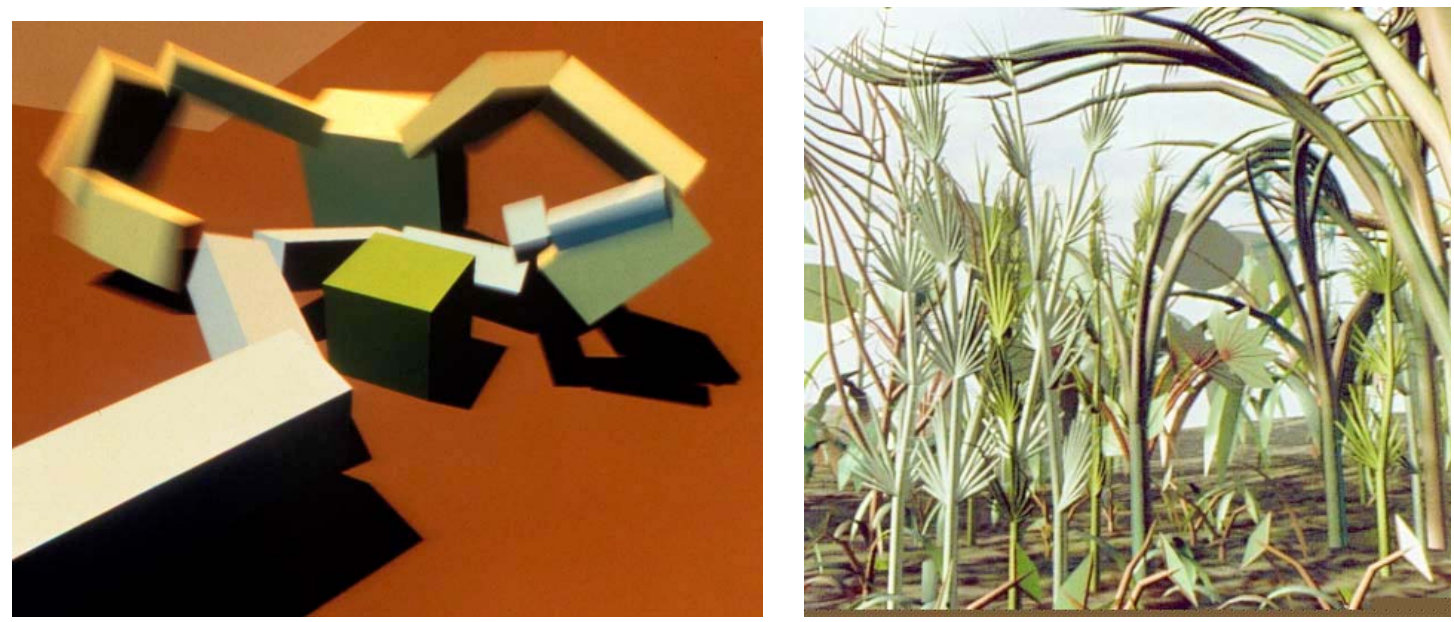

Pictures 1 and 2: Karl Sims' Evolved Virtual Creatures and Panspermia

Both in the Game of Life and in Sims' simulations, creatures evolve based on their relationship to the simulated environment in which they live. Simulations generally simplify complex behaviors and try to model real world features in digital space.

In summary, the development of computers as simulation machines as well as the concept of cyberspace as an immaterial space contributed to reinforce the separation between mind and body inherent to western society. Because art and literature always reflect society (and vice versa) it is common to address the question what is real? in several works. To question what is real? in the age of cyberspace, means we ask: is what we call real physical reality or is it just a simulation running inside our minds?

What would happen if the relationship between inside and outside were altered? This began to happen a few years ago, when we started to see both in arts and in science 
fiction works that connect the physical and the virtual instead of treating them as separate entities.

\section{TOWARD A HYBRID REALITY: MERGING PHYSICAL AND DIGITAL SPACES}

Since the end of the last decade, it has become progressively clearer that physical and virtual spaces are firmly interconnected. Cyberspace is no longer regarded as an isolated space for the mind, but as a place mixed with the physical environment. Actually, the concept of cyberspace itself is now outdated, since it originally means a world of information that exists apart from material space. This hybrid reality has become true partly because the way we connect ourselves to virtual spaces is changing from the imaginary neural implants and computer screens to mobile technology devices and ubiquitous computing. The possibility of being 'always connected,' and to move while connected, contrasts with the act of staring at a computer screen, emphasizing our life in a hybrid reality. When we start to consider virtual space as contiguous to physical space, the question should it simulate the real? is no longer important, because both spaces are merged in the same environment.

In fact, where is the place for the imaginary now that digital space is no longer apart from physical space? This question is clarified once we describe how this connection is being made through technology, art, and literature.

Within the last ten years, experiments that created virtual spaces and creatures isolated from the "real space" were being replaced by works that merge physical and digital spaces. This tendency is true especially in art in works from, for instance, Christa 
Sommerer and Laurent Mignonneau, The Tangible Media Group, and the Aesthetics and Computational Group at the MIT Media Lab. Their works reflect and are influenced by new developments in computer technologies, which contribute to the progressive interconnection between physical and digital spaces. Although still not representing a hybrid reality, their works contributed to define mixed and augmented realities, connecting physical and virtual spaces.

In contrast to Conway's Game of Life and to Sims's Evolved Virtual Creatures, where the simulated system evolves by itself without the need of any external input, Interactive Plant Growing and A-Volve, developed by Christa Sommerer and Laurent Mignonneau, need real people for their evolution. Therefore, these works look for natural interfaces that allow the interaction with virtual environments and deal with the connection between physical and virtual spaces.

Christa and Laurent were actually envisioning what would be the development of the concept of digital space. This transformation started to be considered about ten years ago, but it has been just a few years since we began to feel its effects. However, in experiments like A-Volve, it was clear that there was a virtual space and a physical environment, and that both could influence each other. What happens, then, when these borders are not so well defined anymore? Hence, the main issue about the virtual is no longer how we can inhabit cyberspace, but rather, how do digital spaces change our way of inhabiting the physical world? 

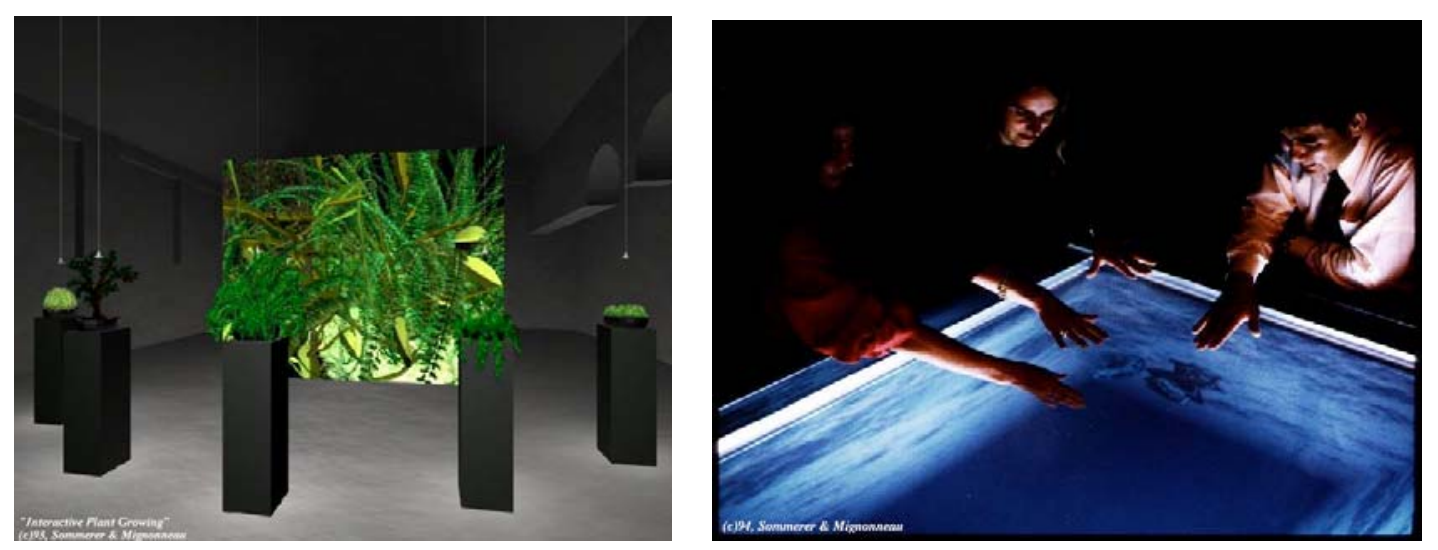

Pictures 3 and 4: Chista Sommerer and Laurent Laurent Mignonneau's Interactive Plant Growing and A-Volve installations

According to Mark Weiser (Weiser 1996), from the Computer Science Lab at Xerox PARC, we are moving toward a third era in the history of computers. The first one he called mainframes, and it was characterized by one computer being shared by lots of people. Also, computers were run mostly by experts. The second phase, in which we were in the last decade, was the personal computing era, where person and machine stared uneasily at each other across the desktop. Each personal computer was meant to belong to one person. Next comes ubiquitous computing, or the age of calm technology, when technology recedes into the background of our lives. The relationship then is going to be many computers to one single person. Thus, ubiquitous computing happens when computers are embedded in our daily lives and no longer part of a distant reality. In this sense, "ubiquitous computing is roughly the opposite of virtual reality. Where virtual reality puts people inside a computer-generated world, ubiquitous computing forces computers to live out here in the world with people" [2] (Weiser 1996). Also, he believes that the future of interface design is going to lead us to an era of invisible interfaces. "A 
less-traveled path I call the 'invisible;' its highest ideal is to make a computer so imbedded, so fitting, so natural, that we use it without even thinking about it."

At this point we can suggest another layer of space that comes after virtual reality and ubiquitous computing. It is the concept of hybrid space. Although all these spatial perceptions can co-exist, there is an intrinsic difference between hybrid reality, mixed reality, augmented reality or virtuality, and virtual reality. Mixed and augmented reality or virtuality are generally connected to the layering of physical/digital data in virtual physical spaces (Milgran 1999). Virtual reality is mostly connected to the idea of inhabiting a computer-modeled world. On the other hand, hybrid spaces are nomadic spaces, created by the constant mobility of users who carry portable devices continuously connected to the Internet. These users are literally carrying the virtual space. Instead of having virtual reality environments through which one can move without changing their physical position, the contemporary nomads of cyberspace in fact move through physical space. Nomadicity is truly a revolutionary change in information technology. It is defined as the systems support provides a rich set of computing and communication capabilities and services to nomads as they move from place to place in a transparent, integrated and convenient form. This new paradigm is already manifesting itself as users travel to many different locations with laptops, PDAs, cellular telephones, and pagers.

Nomadic technologies are mostly used for communication connected to mobility. By doing that, they change our perception of space and time. Wearable computers and transparent interfaces contribute to create a more natural relationship with technology, including them in our everyday life, without the need to really perceive them. Therefore, they become a part of our lives and also a part of our bodies. In addition, nomadic 
technologies both shrink and enlarge the world, changing the relationship between local and global - and between real and imaginary. These connections and differences between what is real and what is imaginary have always played an important role in human history. Philosophy, art, and literature have always tried to address this topic, questioning what the real could be.

Consequently, artists and writers try to address new borders between physical and virtual (real and imaginary), pushing further the limits of technology. Science fiction has always played a critical role in redefining these borders and influencing the future (Neuromancer is a critical example). Recent sci-fi movies address again the question what is real? playing with the relationship between physical and digital spaces. The Matrix (Wachowski 1999) and The Thirteenth Floor (Rusnak 1999) are examples of movies that deal with the connection between the physical and the virtual by placing the virtual either inside our minds and/or together with physical reality.

\section{SCI-FI HYBRID REALITY: ANTICIPATING THE FUTURE}

The movie The Thirteenth Floor begins with the famous quote from Descartes, "I think, therefore I am," suggesting that physical presence is not as important as mental presence, that is, our existence only depends on what we think, on our mind processes. However, as the movie develops, we perceive that human existence also depends on technology and that Descartes's doubt is exactly the point which the movie tries to subvert: Physical presence is indeed important.

The Thirteenth Floor is not the first movie that deals with the idea of simulated spaces by means of philosophical questions. The Matrix has accomplished a similar goal, 
yet in a different way. Early in the film, while the main character Neo (played by Keanu Reeves) works on his computer, we are able to see a book on his desk. It is Simulacra and Simulations, from Jean Baudrillard (Baudrillard 1994), suggesting that the main idea of the film (also what is real?) is constructed through the concept of reality as a simulated space. How can we distinguish reality from simulation, physical from virtual? Similar to Descartes's doubt, the challenge in both movies is to know whether reality is indeed real or simply a simulation running inside computers (and downloaded into human minds). In The Thirteenth Floor, the answer for this question is apparently clear since it is almost evident that there is a simulated world that follows the model of the "real" world. On the other hand, in The Matrix there is no evidence of it until the middle of the film, when we discover that what is "real" is just a simulation for our minds to live in (similar to Neuromancer's idea). Is this simulated world a copy of our "real" world? Or is it something else, a world of data, as in Neuromancer?

The main issue in both movies, however, is how the connection between physical and digital environments is created. In The Matrix there is no connection at all between the two realms. Machines lived in the physical world and humans "lived" in the virtual world. Only some special mortals could have the power to move through both realms and have the awareness of the world's reality. For them was given the power of nomadicity, which allowed them to have the awareness of both physical and digital spaces.

Nevertheless, the majority of human beings had neither consciousness nor movement. They "lived" in a simulated space. Similar to Descartes's idea, their world's appearance had no connection with reality at all. On the other hand, in physical space they could not move, since they were connected to wires and cables. The absence of 
movement is also related to the way people are used to connecting to cyberspace and virtual reality environments. Therefore, what they thought, what they did, was totally determined by the program. People only had the illusion that they could think, but they could not think at all.

Programmed human minds were represented in virtual reality, but their physical bodies were imprisoned in the physical world, connected with cables and wires, being used as batteries. They were condemned to live in virtual space, because of their inability to move in physical space. Hence, the separation between real and simulation is addressed through the absence of the possibility of movement: Immobility prevents the connection between physical and virtual.

Neo, as one of the chosen people to whom were given the power of movement, breaks the enclosure, beginning his real life and connecting virtual and physical. In The Matrix, there's no contact between reality and simulation until Neo makes it. Neo breaks up the disconnection between physical and virtual, setting people free from the simulation.

The idea of simulation as a virtual space that mimics the physical world is not represented literally in the movie. The "matrix" of the movie's title, which is a virtual space created by machines, is not a simulation of the machines' world. It is a re-creation of the humans' world. Therefore, it is similar to a dream space, built for the human mind. This means that the matrix is a simulacrum, [3] with no reference or origin in reality. The matrix creates another reality, which we believe is the real. Hence, it intends to be more real than the real itself because reality is in fact a dark and empty world inhabited by machines. 
In the movie, people no longer enter the virtual space. They belong to it. Something similar happens in The Thirteenth Floor. There are virtual personas that actually live an independent life inside the simulation. Nevertheless, while in The Matrix people who live in the simulation are people who were born in the physical world, The Thirteenth Floor's characters are meant to be completely programmed entities. At first glance, they seem to be like Karl Sims's creatures or cellular automata. However, the development of the movie shows that this first impression is erroneous. Virtual personas are modeled after real people and they can sometimes feel sensations that belong to their models.

The complexity in The Thirteenth Floor arises, then, when we perceive that physical and virtual spaces are so interconnected that one cannot recognize which one is the real and which one is the simulation. Also, opposite to The Matrix, where the absence of movement avoids the connections between physical and virtual, The Thirteenth Floor explores the complexity that happens when movement occurs.

In this movie, the question is no longer what is the real? because real is everything, but what is the connection between physical and virtual spaces? All characters can move through physical and virtual. Not only "real" people are able to download themselves into the simulation, but also virtual personas have the capability of transporting themselves into physical space. Also, avatars (virtual bodies from "real" characters) are able to feel sensations that belong to their models, illustrating that one world influences the other. If avatars are able to live in both spaces, the question what is real? arises again, but with a different connotation, since it is no longer possible to 
separate virtual from physical. Therefore, real must be redefined in order to encompass movement, physical and virtual, creating a hybrid reality.

The Thirteenth Floor deals with complex behaviors that can happen whenever virtual starts to merge with physical. At first glance, avatars seem to have no physical distinction from their models. Nevertheless, they can have completely different personalities, which are sometimes incompatible.

The connection between physical and virtual does not happen only because real characters are able to "jack in" the simulation, but also because virtual personas have the possibility to come to the "real" world, ascending from the simulation. Every time a character is killed while its representation is embodied in the avatar, the digital persona is uploaded to the upper level of the simulation. Characters from the future and past parallel worlds meet in the middle level and the events that arise from these meetings change the whole story. These facts mean that both (or the three) worlds could be connected, but they were not supposed to.

The construction of the 1937 simulation, as well as the 1999 virtual world, were attempts to create imaginary places, which were copies of past realities. However, as the movie develops, the simulations prove to be as real as the "real" world. What is then the distinction between real and imaginary?

Existing virtual worlds, similar to what happens in The Thirteenth Floor, require that the user choose or define an avatar. The virtual world's interface - textual or graphical - connects the user in the physical world to the virtual world. The avatar allows one to "inhabit" the virtual environment. Nevertheless, due to the integration between virtual and physical spaces, the critical issue is no longer creating avatars and 
playing with identities, since we can be anywhere at the same time. Mobility has replaced identity matters. Moreover, since the main concern about digital space is no longer space itself but its connections, body issues are also replaced by speed issues. Concern changes from how should people be represented in cyberspace? to how long does it take to access other points of the network/world?

Science fiction has contributed to bring up a world where this thin layer that separates the user from the virtual environment becomes transparent. In The Matrix, it is possible to enter the simulation through the user's own body. Therefore, the user becomes its own avatar, and the boundaries between physical and virtual are softened. In addition, The Thirteenth Floor deals with what could happen if we could go in and out of virtual spaces, having them influence our lives in physical space. Yet people no longer enter the virtual space. The concept of virtual space has changed, being included in physical reality. Now one can move around the city connected $24 / 7$ to virtual spaces via his or her cell phone. It is possible to connect people on the other side of the world very quick and also to download and upload any content to the Internet in real-time.

Nowadays the question what is real? starts to have another meaning, since the physical is being directly influenced by the digital. In the era of personal computing and virtual reality, we questioned the real due to the existence of another space, virtual, which could be modeled after the physical space, but could also be imaginary. Yet in the era of hybrid reality, the question what is real? has been used to affirm that there are no longer two different realities (virtual and physical). There is only one that contains in itself both the physical and the virtual. In addition, virtual also obtains another meaning, since the virtual as a discrete space is vanishing. 
When we can be everywhere at the same time, space becomes time and our sense of real is augmented. Consequently, due to the hybrid space that emerged with nomadic technologies, a new dichotomy between real and imaginary arises. If before the virtual could be synonymous to the imaginary, now imaginary spaces are included in physical space. But in fact, what is real?

\section{REFERENCES}

- Jean Boudrillard. Simulacra and Simulation. The Body, in Theory: Histories of Cultural Materialism, Ann Arbor, University of Michigan Press, 1994.

- Gary William Flake, The Computational Beauty of Nature - computer explorations of fractals, chaos, complex systems, and adaptation, Cambridge, The MIT Press, 2000.

- William Gibson, Neuromancer, New York, Ace Books, 2000.

- Katherine Hayles, How we Became Posthuman - virtual bodies in cybernetics, literature, and informatics, Chicago, The University of Chicago Press, 1999.

- Paul Milgran, Herman Colquhoun Jr., 'A taxonomy of real and virtual world display integration'. Mixed reality: merging real and virtual worlds. Yuichi Ohta, Hideyuki Tamura (ed.), Tokyo, Ohmsha / Secaucus, New Jersey, Springer-Verlag New York, c1999. p. 5-28.

- Janet Murray, Hamlet on the Hollodeck - the future of narrative in cyberspace Cambridge, The MIT Press, 1999.

- Sherry Turkle, Life on the Screen - Identity in the age of the Internet, New York, Simon \& Schuster, 1995. 
- Robert Wright, “A Finely Mottled Universe”, Chapter 3, Three Scientists and Their Gods - Looking For A Meaning In The Information Age, New York, Harper \& Row, 1989, pp. 21-27.

\section{WEB REFERENCES:}

- aesthetics + computation group :: mit media laboratory. (Accessed: 2002, November 16), Available: http://acg.media.mit.edu.

- Karl Sims (Accessed: 2002, November 16), Karl Sims Home Page, http://www.genarts.com/karl/index.html (2002)

- Christa Sommerer and Laurent Mignonneau, L. (Accessed: 2002, November 16), Home Page Christa Sommerer \& Laurent Mignonnuau, Available: http://www.mic.atr.co.jp/ christa/

- Tangible Media Group. MIT Media Lab. (Accessed: 2002, November 16), Available: http://tangible.media.mit.edu/ .

- Mark Weiser, (Accessed: 2002, November 16), Xerox Palo Alto Research Center -- Sandbox Server, 1996), Available:

http://www.ubiq.com/hypertext/weiser/UbiHome.html.

\section{FILM REFERENCES:}

- Josef Rusnak, The Thirteenth Floor, USA, 1999.

- Larry \& Andy Wachowski Brothers, The Matrix, USA, 1999.

\section{NOTES}

${ }^{1}$ Concept created by William Gibson in 1985. Cf. GIBSON 2000. 
${ }^{2}$ Weiser, Mark. Ubiquitous Computing. March 17, 1996.

<http://www.ubiq.com/hypertext/weiser/UbiHome.html> (January 12, 2004)

${ }^{3}$ According to Baudrillard's concept of simulacra.

PICTURE CREDITS

Picture 1: Image from "Virtual Creatures" courtesy of Karl Sims (c) 1994.

Picture 2: Image from "Panspermia" courtesy of Karl Sims (c) 1990.

Picture3: Interactive Plant Growing

(c) 92, Christa Sommerer \& Laurent Mignonneau

interactive computer installation

collection of the Mediamuseum at the ZKM Karlsruhe

Picture 4: A-Volve

(c)94, Christa Sommerer \& Laurent Mignonneau

interactive computer installation

supported by ICC-NTT Japan and NCSA, USA 